\title{
Optical collisions of cold, metastable helium atoms
}

\author{
H.C. Mastwijk, J.W. Thomsen, P. van der Straten and A. Niehaus \\ Debye Institute, Department of Atomic- and Interface Physics, \\ Utrecht University, P.O. Box 80.000, 3508 TA Utrecht, The Netherlands
}

We have studied the optical collisions of cold, metastable helium atoms in a magneto-optical trap. We have detected the rate of Penning and associative ionization of two metastable helium atoms at a temperature of $1 \mathrm{mK}$ with and without nearly resonant light. We find that the associative ionization rate is increased with more than a factor 20 due to the presence of the light field. The absolute ionization rate near resonance of $(1.9 \pm 0.8) \times 10^{-9} \mathrm{~cm}^{3} / \mathrm{s}$ for the optical collision differs two orders of magnitude with the rate reported by Bardou et al. (Europhys. Lett. 20, 681 (1992)). Our experimental findings are in good agreement with theory. We present a simple, semi-classical model, which accounts for all the observed features.

Pacs Numbers: 32.80.Pj, 33.80.Eh, 34.50.Rk

Due to the breakthrough of laser cooling techniques it is nowadays possible to study collision processes at ultra-low temperatures [1]. The study of optical collisions, where during the collisions of two cold atoms a photon is absorbed, has been very successful. It has lead to a wealth of experimental data on the long range interactions in these systems and has been shown to be complementary to molecular spectroscopy [2]. While in the latter case one probes the molecular structures at short range, in the former case one obtains information on the interaction taking place at long range and is able to observe the molecular complex during the reaction.

Optical collisions can be induced by irradiation of light detuned below atomic resonance. The transition energy at long range is lowered due to the dipole-dipole attraction $C_{3} / R^{3}$ in the first excited state and the excitation point $R_{c}$ can be selected by the detuning of the light. The study of the ionization rate as a function of the detuning provides information on the dynamics and ionization of the molecular excited states involved. In general, the ionization rate will increase if the laser is tuned closer to resonance, since the number of atomic pairs that can be excited will increase by increasing $R_{c}$. However, once the detuning becomes too small the ionization rate will decrease for two reasons. First of all, the atomic pairs are excited at such a large distance, that spontaneous emission during the time that the molecular system reaches small internuclear distances leads to a "switching-off" of the attractive $1 / R^{3}$-potential, thus prohibiting a close collision in which ionization can occur. Secondly, the attractive potential becomes too weak compared to the relative kinetic energy, so that absorption does not lead to a close collision.

We have studied optical collisions using cold, metastable $\mathrm{He}\left(2{ }^{3} \mathrm{~S}\right)$ atoms. Although optical collisions have already been studied experimentally in several systems [3], the $\mathrm{He}\left(2^{3} \mathrm{~S}\right)$-system is in many aspects different from all other systems. $\operatorname{He}\left(2^{3} \mathrm{~S}\right)$ is a prototype system with only one electron active and no hyperfine structure. This makes it possible to obtain accurate potential curves for the $\mathrm{He}\left(2^{3} \mathrm{~S}\right)-\mathrm{He}\left(2^{3} \mathrm{~S}\right)$ system, which have been published recently [4]. It is therefore possible to obtain a direct comparison on an absolute scale between experiment and theory for this system. Another difference becomes clear, if one considers two important parameters in optical collisions, which identify the reaction dynamics. The first parameter, $R_{\tau}=v \tau$, is the average distance an atom with velocity $v$ travels in the excited state with lifetime $\tau$, before it decays spontaneously. The second parameter, $R_{\lambda}=\lambda / 2 \pi$, is the characteristic distance, at which retardation effects in the molecular complex become important. In all cold collision systems studied so far $R_{\tau}$ is two orders of magnitude smaller than $R_{\lambda}$. However, for the $\mathrm{He}\left(2^{3} \mathrm{~S}\right)$ system at $1 \mathrm{mK}, v=3 \mathrm{~m} / \mathrm{s}, \tau=98 \mathrm{~ns}$ and $\lambda=1.083 \mu \mathrm{m}$, so $R_{\tau}=5600 a_{0}$ and $R_{\lambda}=3300 a_{0}$. Since $R_{\lambda}$ is of the same order of magnitude and even larger than $R_{\tau}$, one expects that the reaction dynamics will be different compared to the other systems. Finally, if the atoms react on the $S-S$ potential, only the lowest order partial wave $(\ell=0)$ is expected to contribute to the scattering cross section, since the kinetic energy in the reaction is insufficient for atoms to cross the rotational barrier for $\ell \neq 0$. In the optical S-P collision, where the atoms react on the $C_{3} / R^{3}$ potential, $\approx 10$ partial waves contribute to the cross section and the reaction can be described semiclassical. By absorption of a photon we are thus able to switch from the quantum-mechanical regime to the classical regime without changing the collision energy.

In the experiment metastable helium atoms are produced in a discharge source cooled by liquid nitrogen following the design of Kawanaka et al. [5]. The atoms are slowed down over a distance of 2 meter using the technique of Zeeman-compensated slowing [6] and loaded into a magneto-optical trap (MOT). The light for both slowing and trapping is produced by diode lasers manufactured especially for the $2^{3} \mathrm{~S} \rightarrow 2^{3} \mathrm{P}$ transition at $1.083 \mu \mathrm{m}$ [7]. Since the loading rate of the metastable atoms in the trap is small compared to typical rates for alkali metals, the trap is operated in a regime, where the capture velocity is large. This has been realized by using a large detuning $(\delta=-25 \mathrm{MHz})$ and a high inten- 
sity of the trapping laser (saturation parameter $s_{0}=50$ per beam). Typical values for our trap are $1 \times 10^{5}$ for the number of atoms and $2.5 \times 10^{7}$ atoms $/ \mathrm{cm}^{3}$ for the density. The temperature of the atoms is $1.1 \mathrm{mK}$, as determined by a time-of-flight (TOF) technique. Here the atoms are released from the MOT and the time of arrival of the atoms on a set of micro-channel plates is detected. The trap density and temperature are in agreement with a simple model for the operation of the MOT at such large detunings taking into account only the Doppler theory for cooling and trapping [8].

Ions produced in the MOT are accelerated with a small voltage towards a quadrupole mass-spectrometer, which selects the ions on their mass. We find that most of the ions are produced by Penning ionization (PI) of background gas atoms on $\mathrm{He}\left(2^{3} \mathrm{~S}\right)$. Since $\mathrm{He}\left(2^{3} \mathrm{~S}\right)$ has a high internal energy, it can Penning ionize almost all atoms and molecules. We have identified that the major inelastic trap loss is by $\mathrm{PI}$ of $\mathrm{H}_{2} \mathrm{O}$ on $\mathrm{He}\left(2^{3} \mathrm{~S}\right)$, even at a background pressure of $4 \times 10^{-7} \mathrm{~Pa}$. This is due to the large PI cross section for this process of $70 \AA^{2}$ [9]. Ions produced with mass $4\left(\mathrm{He}^{+}\right)$and mass $8\left(\mathrm{He}_{2}^{+}\right)$are also produced and these ions can only be produced in intraMOT collisions, since the density of $\mathrm{He}\left(2^{3} \mathrm{~S}\right)$ atoms in the background is orders of magnitude smaller than the density in the trap. Thus by mass selection we can uniquely discriminate between collisions of $\mathrm{He}\left(2^{3} \mathrm{~S}\right)$ with the background gas atoms, which occur at thermal energies, and intra-MOT collisions, which take place at $1 \mathrm{mK}$. The detection efficiency of the mass spectrometer is calibrated by detecting the total number of ions produced, irrespective of their mass, with a second detector mounted opposite to the mass spectrometer, where all ions are detected with a large efficiency by micro-channel plates.

To detect the ion rate as a function of the detuning of the laser from atomic resonance we change the frequency of the MOT laser during a period of $60 \mu \mathrm{s}$. This period is sufficiently short, such that the spatial distribution of the atoms in the MOT is not altered by the absence of the trapping laser light, but still long compared to the lifetime of the $\mathrm{He}\left(2^{3} \mathrm{P}\right)$-state. In this period the laser is first detuned to resonance and then ramped with a fixed rate far below resonance by changing the current through the diode. The $\mathrm{He}_{2}^{+}$signal as a function of time is recorded and afterwards converted to laser detunings by using the scan rate and the known position of the laser frequency for trapping. Note, that since we are switching the frequency of the MOT-laser beams, the saturation parameter of the laser inducing the ionization is very high, namely $s_{0}=300$ (50 per beam).

Data recorded for several different runs are shown in figure 1. One observes that the ionization rate increases dramatically if the laser is tuned close to resonance. When the laser is detuned slightly below resonance we observe a maximum in the ionization rate. The overall increase in the ionization signal due to the presence of the laser light is about a factor 20 .

In a second experiment we have detected the fraction of ions produced as $\mathrm{He}_{2}^{+}$compared to the total number of ions produced in intra-MOT collisions. In the PI process the excess of internal energy of the two metastable atoms is carried away by the ejected electron. The outcome of the process for the heavy particles depends on the amount of energy carried away by the emitted electron. If the electron energy is more than the excitation energy of the two metastable atoms minus the ionization potential, then the heavy particles become bound and a molecular ion $\mathrm{He}_{2}^{+}$is formed. Otherwise, an atomic $\mathrm{He}^{+}$ion is produced. Due to the mass selection in our experiment we can discriminate between these two reaction channels.

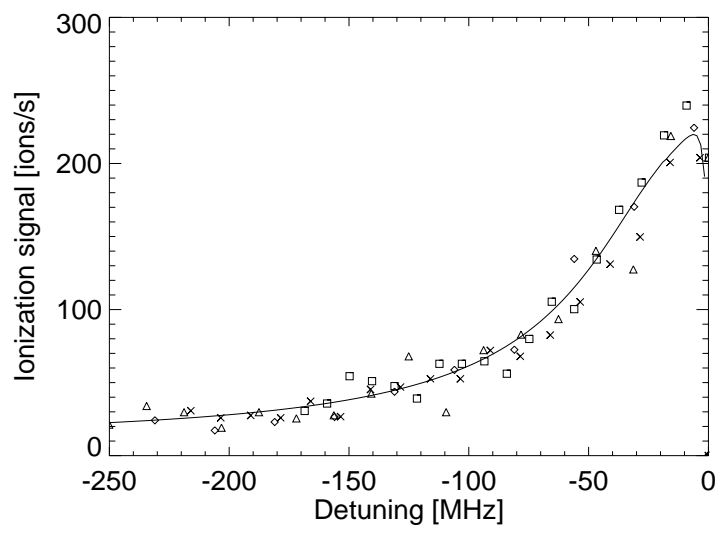

FIG. 1. Ionization signal of $\mathrm{He}_{2}^{+}$-ions produced in optical collisions as a function of the detuning of the trapping laser. The saturation parameter of the laser beams is $s_{0}=50$ per beam. The symbols refer to the experimental results, where we used a different symbol for each run. The solid line is the result of our semi-classical model.

The fraction of $\mathrm{He}_{2}^{+}$ions is measured by changing the trapping laser in a period of $20 \mu \mathrm{s}$, but the detuning of the laser frequency was fixed during this period. By switching the mass spectrometer between mass 4 and 8 we can obtain the $\mathrm{He}_{2}^{+}$fraction as a function of frequency and we find close to resonance a fraction of $0.16 \pm 0.02$, whereas far from the resonance the fraction becomes $0.030 \pm 0.003$, where the error bars refer to the statistical uncertainty. In the latter case we only have $\mathrm{He}\left(2^{3} \mathrm{~S}\right)-\mathrm{He}\left(2^{3} \mathrm{~S}\right)$ collisions and the measured fraction is in agreement with the theoretical value of 0.028 calculated using the potential and width function of Müller et al. [4]. The vastly different fractions on- and off-resonance is a clear indication that $\mathrm{PI}$ for the S-S and S-P reactions occur through different potentials, which is to be expected due to the long lifetime of the excited state. One complication in the analysis is that the detection efficiency of $\mathrm{He}^{+}$and $\mathrm{He}_{2}^{+}$ions is not identical, since the molecular ions are produced with nearly zero kinetic energy, whereas the atomic ions obtain kinetic energy in the fragmentation of the reaction 
complex. We have estimated this reduction of the detection efficiency by calculating trajectories of the atomic ions through the setup and corrected the fractions for this effect.

Using the values for the density and the volume of our trap and the measured fraction of $\mathrm{He}_{2}^{+}$ions we find a total ionization rate in the absence of light of $(2.7 \pm 1.2) \times 10^{-10}$ $\mathrm{cm}^{3} / \mathrm{s}$. Since we operate the MOT with a large intensity of the trapping laser the excitation probability $\Pi_{e}$ of the atoms close to resonance becomes $\Pi_{e}=0.5$. From this we can derive a total ionization rate for $\mathrm{He}\left(2^{3} \mathrm{~S}\right)-\mathrm{He}\left(2^{3} \mathrm{P}\right)$ or $\mathrm{S}-\mathrm{P}$ reactions of $(1.9 \pm 0.8) \times 10^{-9} \mathrm{~cm}^{3} / \mathrm{s}$. The last value is a factor 100 smaller compared to the value of $1 \times 10^{-7}$ $\mathrm{cm}^{3} / \mathrm{s}$ reported by Bardou et al. [10].

To compare our value for $\mathrm{He}\left(2^{3} \mathrm{~S}\right)-\mathrm{He}\left(2^{3} \mathrm{~S}\right)$ ionizing reactions with theory we have carried out quantummechanical scattering calculations using the potentials of Müller et al. [4]. To include the ionization process we have added the complex part $\Gamma(R)$ to the potential in the partial wave analysis, which is given for the $\mathrm{He}\left(2^{3} \mathrm{~S}\right)$ $\mathrm{He}\left(2^{3} \mathrm{~S}\right)$ system by the same authors. We carried out calculations for the $1^{1} \Sigma_{g}^{+}$and $1^{3} \Sigma_{u}^{+}$state and find a total ionization rate at $1 \mathrm{mK}(\approx 100 \mathrm{neV})$ of $7.3 \times 10^{-11}$ $\mathrm{cm}^{3} / \mathrm{s}$. From the comparison between this value and our experimental result we can infer, that we have made a correct estimate of the density and volume of our trap. Since the lowest partial wave for the uneven $1^{3} \Sigma_{u}^{+}$state in this boson system is $\ell=1$ and we are in the regime of $s$-wave scattering, the elastic cross section in the $1^{3} \Sigma_{u}^{+}$ state nearly vanishes. However, the ionization rate corresponding to this state does not vanish, which is to be expected for an inelastic process [11], and contributes in our case for $\approx 1 / 3$ to the total ionization rate.

In order to explain the measured absolute rate for S-P collisions and the measured line shape, one has to describe the optical collision process. Although several theoretical models exist [12], we will describe our own model here, since it reveals in a simple way the essence of the process without having to rely on a complex analysis. In our model we assume that a pair of atoms becomes excited at the Condon point $R_{c}=\left(C_{3} / \hbar \delta\right)^{1 / 3}$, where the excitation probability $P_{\text {exc }}$ is given by the Landau-Zener transition probability $P_{\text {exc }}=1-\exp (-\pi \Lambda)$, with [13]

$$
\Lambda=\frac{2 \hbar \Omega^{2}}{\alpha v_{\mathrm{rad}}} .
$$

Here $\Omega$ is the Rabi-frequency, $\alpha=3 C_{3} / R_{c}^{4}$ is the gradient of the potential at $R_{c}$ and $v_{\text {rad }}$ the radial velocity. The Rabi-frequency is related to the knwon saturation parameter $s_{0}$ by $s_{0}=2 \Omega^{2} / \Gamma^{2}$. The number of atomic pairs $\dot{N}$ crossing the Condon sphere is given by the classical pair distribution $\dot{N}=4 \pi R_{c}^{2} n v_{\text {rad }}$ [14], with $n$ the density of the atoms and $v_{\text {rad }}$ the radial velocity. The classical trajectory of the molecular complex in the excited state is calculated for the different, thermally dis- tributed, initial velocities. For each trajectory it is determined if the reaction proceeds to short range, where the reaction takes place, and only these close collisions are considered. The survival probability of the complex becomes $P_{\text {surv }}=\exp (-\Gamma t)$, where $t$ is the elapsed time between excitation and reaction. Note, that our model becomes identical to the GP-model [14] for the case of large detuning and low intensity. However, we extend on it by including a dynamical excitation probability for our case of high intensity and by including a numerical calculation of the trajectory including different initial conditions. Also Suominen et al. [15] have extended the GP-model to include the dynamical excitation probability into the model. However, their model does not include the calculation of the trajectories, which is essential to obtain agreement for small detunings, where the attractive potential is of the same order as the kinetic energy of the molecular complex.

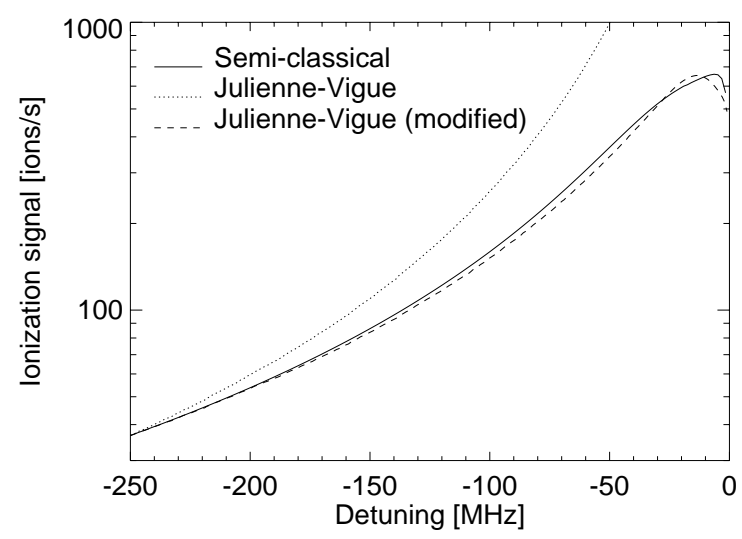

FIG. 2. Comparison between our semi-classical model (solid line), as discussed in the text, and the models published in the literature. In the plot we have included the results for the JV-model (dotted line) and the modified JV-model (dashed line), where we have replaced the quasi-static excitation probability with the dynamical Landau-Zener transition rate.

The result of our analysis is given in figure 1 . In this analysis we have averaged the results over all attractive molecular states, that asymptotically connects to the $2^{3} \mathrm{~S}-2^{3} \mathrm{P}_{2}$ asymptote. For each of these states $\beta$ we have determined the $C_{3, \beta}$ coefficient and the decay rate $\Gamma_{\beta}[16]$. In addition, we have determined what fraction of these states connect to the $1^{1} \Sigma_{g}^{+}$and $1^{3} \Sigma_{u}^{+}$state at short range, since only these two states can Penning ionize. The agreement between the measured and the theoretical lineshape is very good over the whole range of detunings. This shows, that our simple model applied to the $\mathrm{He}\left(2^{3} \mathrm{~S}\right)$ system completely describes the dynamics of the reaction. One important aspect of this system is that, since $R_{\tau}$ is so large, the survival probability does not play any significant role, and the correct lineshape 
is already obtained by a proper description of the excitation process of the molecular complex by the LandauZener transition probability and by taking into account the evolution of the system in the excited state.

In order to compare our model with theoretical models already discussed in the literature we have carried out calculations based on the modified JV-model [16], where we have replaced their quasi-static excitation probability by the dynamic Landau-Zener probability. The result of this analysis is shown in figure 2 together with the result of our semi-classical model. The agreement between the two models is excellent, apart from a small range around zero detuning. The good agreement is to be expected if one considers that both models describe the same dynamics in the molecular complex. In our model this is done semi-classical, whereas the JV-model relies on a quantum-mechanical description. Since the number of partial waves in the optical collision is large $(\approx 10)$, both methods should work. To show that the quasi-static approximation does not work in our case of high intensities we have calculated the lineshape based on the original JV-model and indicated the results in figure 2. Already at detunings of the order of $100 \mathrm{MHz}$ one can observe the discrepancy between the quasi-static models and the dynamical one. Since the agreement between the data and the dynamical models is excellent, it is clear that these models describe the excitation process most accurately.

Both our semi-classical analysis and the quantummechanical analysis based on the JV-model provide absolute rate constants. For the semi-classical analysis we find a rate constant for S-P collisions close to resonance of $1.1 \times 10^{-9} \mathrm{~cm}^{3} / \mathrm{s}$. This value is consistent with the experimental result of $(1.9 \pm 0.8) \times 10^{-9} \mathrm{~cm}^{3} / \mathrm{s}$. Therefore, we must conclude that the rate constant measured by Bardou et al. [10] of $1 \times 10^{-7} \mathrm{~cm}^{3} / \mathrm{s}$ is two orders of magnitude too large. In their experiment they have probably underestimated the density, which they have determined by detecting the image of the MOT cloud with a CCDcamera. Our density is based on a direct detection of the number of atoms in the TOF-analysis combined with an estimate of the trap radius from model calculations, which is closely related to the temperature.

The special situation of detecting reaction products with light-on and light-off allows for a direct comparison between the rate constants for $\mathrm{S}-\mathrm{P}$ and $\mathrm{S}-\mathrm{S}$ collisions. Note, that the first type of reaction can be described semi-classical, whereas the second type calls for a fully quantum-mechanical treatment. This is one of the special features of ultra-cold collisions in the presence of a light field and is a valuable asset for testing theoretical models. Since the ratio does not depend on the density and volume of the trap it is determined with a much higher accuracy than the absolute rates separately. In the experiment we find a ratio of 7.2 , whereas the models predict a ratio of 15.0. Regarding the uncertainty of the $\mathrm{S}-\mathrm{S}$ rate, which is very sensitive in the case of ultra- cold collisions to the detailed shape of the potential, the agreement is satisfactory.

The large ionization rate at resonance is due to the large distance the $\mathrm{He}\left(2^{3} \mathrm{~S}\right)$-system can travel in the excited state. In all other systems studied so far this distance is small and in these systems the rate goes to zero close to resonance. This is not the case for the $\mathrm{He}\left(2^{3} \mathrm{~S}\right)$ system and atoms excited at large internuclear distance are still approaching each other in the excited state. The $\mathrm{He}\left(2^{3} \mathrm{~S}\right)$-system is therefore the ideal system to study the transition from the molecular regime, where the excitation is localized at the Condon radius, to the atomic regime, where the excitation is spread over a very large range of internuclear distances. At these large distances retardation effects play an important role and a careful study of the ionization rate close to resonance should reveal such retardation effects directly.

In conclusion, we have investigated optical collisions of cold, metastable helium atoms. The dynamics of the $\mathrm{He}\left(2^{3} \mathrm{~S}\right)$ system is different compared to other systems studied so far, since the lifetime of the excited state is rather large and the mass is small. We show that the results obtained can be satisfactorily described by a simple semi-classical model, which accounts for all the features observed. Since Penning ionization can take place both from the S-S and S-P potential, it is possible to compare the ionization rates for these two channels. In the first case the reaction can only be described fully quantummechanical, whereas is the second case a semi-classical description is sufficient. The agreement between theory and experiment for the ratio between the rates for S-P and S-S reactions is rather good.

This work has been supported by the "Stichting voor Fundamenteel Onderzoek der Materie (FOM)", which is financially supported by the "Nederlandse organisatie voor Wetenschappelijk Onderzoek (NWO)" and JWT is supported by the European Union's TMR programme under contract number ERB4001GT95292.

[1] H. Metcalf and P. van der Straten, Phys. Rep. 244, 203 (1994).

[2] P.D. Lett, P.S. Julienne, W.D. Phillips, Rev. Phys. Chem. 46, 423 (1995)

[3] M.G. Peters et al., Phys. Rev. A 50, R906 (1994), H. Katori and F. Shimizu, Phys. Rev. Lett. 73, 2555 (1994), M. Walhout et al., Phys. Rev. Lett. 74, 506 (1995), P.A. Molenaar et al., Phys. Rev. Lett. 77, 1460 (1996), J.J. Blangé et al., Phys. Rev. Lett. 78, 3089 (1997).

[4] M.W. Müller, A. Merz, M.-W. Ruf, H. Hotop, W. Meyer and M. Movre, Z. Phys. D 21, 89 (1991).

[5] J. Kawanaka, M. Hagiuda, K. Shimizu, F. Shimizu and H. Takuma, Appl. Phys. B 56, 21 (1993). 
[6] P.A. Molenaar, P. van der Straten, H.G.M. Heideman, H. Metcalf, Phys. Rev. A 55, 605 (1997).

[7] J.S. Major Jr. and D.F. Welch, Electr. Lett. 29, 2121 (1993).

[8] H.C. Mastwijk, thesis Utrecht 1997 (unpublished).

[9] W. Allison and E.E. Muschlitz, J. of. Elec. Spectr. 23, 339 (1981).

[10] F. Bardou, O. Emile, J.-M. Courty, C.I. Westbrook and A. Aspect, Europhys. Lett. 20, 681 (1992).

[11] L.D. Landau and E.M. Lifschitz, Quantum Mechanics, 2nd ed., Pergamon, Oxford, 1965.

[12] K.-A. Suominen, J. Phys. B 29, 5981 (1996).

[13] M.J. Holland, K.-A. Suominen and K. Burnett, Phys. Rev. A 50, 1513 (1994).

[14] A. Gallagher, D.E. Pritchard,Phys. Rev. Lett. 63, 957 (1989)

[15] K.-A. Suominen, K. Burnett, P.J. Julienne, M. Walhout, U. Sterr, C. Orzel, M. Hoogerland, and S.L. Rolston, Phys. Rev. A 53, 1678 (1996).

[16] P.S. Julienne and J. Vigué, Phys. Rev. A 44, 4464 (1991). 